\title{
Estandartes carnavalescos alagoanos: uma análise dos seus elementos gráficos
} Carnival banners from Alagoas: an analysis of its graphic elements

\author{
Felipe D. Albuquerque, Mariana Hennes
}

estandartes carnavalescos, cultura popular, catalogação, linguagem gráfica

\begin{abstract}
Os estandartes de carnaval são artefatos culturais de produção artesanal que funcionam, na contemporaneidade, identificando grupos momescos de rua. Eles são detentores de grande riqueza iconográfica, por possuírem, em suas configurações, uma variada gama de cores, formas, materiais, composições e elementos gráficos que representam uma importante parcela da cultura material local, cujo conteúdo ainda encontra-se inexplorado, do ponto de vista do design. Nesse sentido, o objetivo desse estudo foi registrar, catalogar e descrever os elementos e atributos gráficos presentes nos estandartes alagoanos de carnaval, a partir de uma metodologia quanti-qualitativa, adaptada às necessidades da pesquisa. Os resultados encontrados permitiram traçar um panorama dos elementos da linguagem gráfica presente em vinte e quatro artefatos. Assim, conclui-se que há uma grande produção de estandartes feitos por artistas com especialidade técnica no estado de Alagoas, elaborados a partir de uma grande variedade de materiais e elementos gráficos, que possuem similaridades entre si.
\end{abstract}

carnival banners, popular culture, cataloging, graphic language

Carnival banners are cultural artefacts of artisan production that work, in the contemporaneity, identifying street carnival groups. They are holders of great iconographic richness, because they have, in their configurations, a varied range of colors, forms, materials, compositions and graphic elements that represent an important part of the local material culture, the content of which is still unexplored, from the point of view of design. In this sense, the objective of this study was to record, catalog and describe the elements and graphic attributes present in the Alagoas carnival standards, based on a quantitativequalitative methodology was adopted, adapted to the needs of the research. The results found allowed us to outline the elements of the graphical language of twenty four artifacts. Thus, it is concluded that there is a great production of banners made by artists with technical expertise in the state of Alagoas, elaborated from a wide variety of materials and graphic elements, which have similarities among themselves.

\section{Introdução}

A celebração do carnaval é, no Brasil, um dos momentos de maior adesão popular, sendo essa festa considerada parte da sua identidade cultural. Segundo o antropólogo Cavalcanti (2014), as diferentes regiões brasileiras brincam o carnaval de formas distintas, tanto nas dimensões socioeconômicas quanto simbólicas. Assim, cada parte do país apropriou-se de elementos culturais próprios, imputando, ao festejo, elementos locais da cultura popular.

O carnaval alagoano se assemelha, em alguns aspectos, ao carnaval de rua dos vizinhos pernambucanos. A folia é marcada por ritmos folclóricos diversos (como o frevo, o maracatu e o caboclinho); e por algumas manifestações peculiares, como os bonecos gigantes e as fantasias dos foliões - geralmente com teor cômico. Estes se aglomeram nas ruas e desfilam com uma configuração semelhante aos cortejos religiosos Europeus.

De acordo com Silva (2016), além da estrutura física - forma em que se organiza o andor -, um dos elementos das procissões quaresmais europeias que mais integrou os costumes carnavalescos brasileiros foi o pendão: bandeira de grandes proporções, descendente das peças utilizadas nas corporações da Idade Média e do Brasil colônia, levada sempre à frente do cortejo.

Assim, no Brasil, a fim de apresentar a identidade dos grupos brincantes no carnaval de rua, esse antigo artefato de sinalização se fez presente também no carnaval. Os estandartes constituem artefatos ricos em adornos, confeccionados a partir de processos de produção

Anais do 8 $\mathrm{CIDI}$ e 8 CONGIC

Guilherme Santa Rosa; Cristina Portugal (orgs.)

Sociedade Brasileira de Design da Informação - SBDI

Natal | Brasil | 2017

ISBN 978-85-212-1305-5
Proceedings of the $8^{\text {th }} \mathrm{CIDI}$ and $8^{\text {th }}$ CONGIC

Guilherme Santa Rosa; Cristina Portugal (orgs.)

Sociedade Brasileira de Design da Informação - SBDI

Natal | Brazil | 2017

ISBN 978-85-212-1305-5 
passados de geração em geração, e elaborados seguindo uma composição gráfica peculiar, que envolve desde seu formato até a escolha e diagramação dos elementos informacionais/decorativos (figura 1).

Figura 1: Estandartes desfilando no bloco Pinto da Madrugada, em Maceió-AL (fonte: autoral).



\section{Análise dos estandartes sob a ótica do design}

Considerando a representatividade dos estandartes nos festejos carnavalescos locais e a riqueza iconográfica presente em suas composições, procurou-se aproximar esse nicho da cultura popular aos estudos acadêmicos no campo do design, no intuito de não só reconhecê-lo e valorizá-lo, mas também de entender como se dá a composição gráfica destas peças, identificando padrões estéticos e procurando formas de apropriação e divulgação destes elementos na contemporaneidade.

Estimado seu valor histórico e cultural, muitos deles fazem parte dos acervos dos museus de antropologia e cultura popular no Brasil, e embora sua riqueza iconográfica seja reconhecida em diversas instancias sociais, pesquisas sobre esses artefatos ainda são muito incipientes em instituições acadêmicas.

Sob o ponto de vista do design, os elementos imagéticos dos estandartes chamam tanta atenção quanto os elementos textuais, que trazem o nome e o ano de criação do bloco. No entanto, para fins didáticos, do ponto de vista do design da informação, a análise dos elementos compositivos aconteceu de forma fatorada.

Essa desconstrução serviu como premissa das análises gráficas realizadas, essenciais a essa pesquisa acadêmica. Para construir esse estudo, foi preciso categorizar e classificar seus elementos, fatorando o objeto de estudo em camadas (figura 2). 
Figura 2: Esquema em perspectiva explodida para compreensão da divisão das camadas (fonte: autoral).

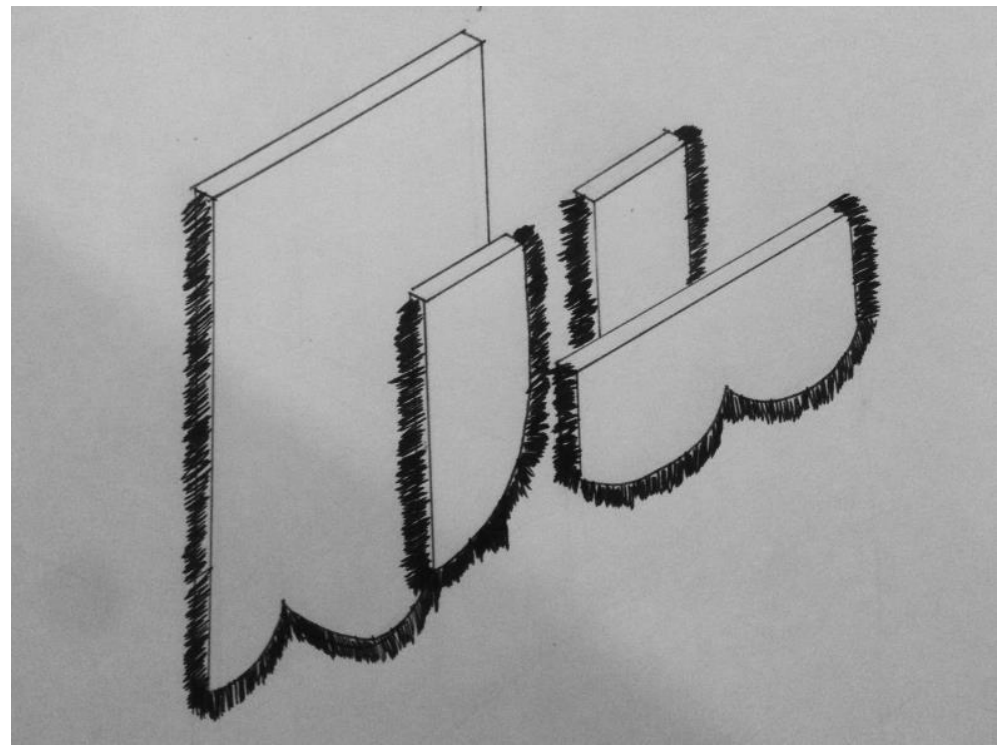

Segundo Faria \& Souto (2014) os estudos de Twyman (2002) contribuíram para a análise da linguagem visual, denominada por este como linguagem gráfica (LG). Ele define gráfico como aquilo que é desenhado ou feito visível em resposta a decisões conscientes, e linguagem o veículo de comunicação. Assim, a LG é composta por marcas produzidas a mão e/ou por máquinas com o intuito de comunicar uma mensagem, e seus elementos podem ser classificados como verbais, pictóricos e esquemáticos, conforme demonstrado na tabela 1:

Tabela 1: Adaptação do esquema de elementos da Linguagem Gráfica segundo Twyman (2002) (fonte: autoral).

\begin{tabular}{l|l} 
Tipo de elemento & Definição \\
\hline Verbal & Palavras e números \\
\hline Pictórico & $\begin{array}{l}\text { Qualquer representação feita a mão ou a máquina } \\
\text { que se relaciona, mesmo que distante, a coisas } \\
\text { existentes ou imagináveis }\end{array}$ \\
\hline Esquemático & $\begin{array}{l}\text { Tudo que, decididamente, não for verbal (caractere } \\
\text { alfabético ou numérico) ou pictórico. }\end{array}$ \\
\hline
\end{tabular}

\section{Metodologia de pesquisa}

Utilizando como referência a metodologia desenvolvida por Hennes (2012), a presente pesquisa se destrinchou em cinco etapas distintas: (a) composição do universo de análise a partir de registros fotográficos do objeto de estudo, (b) seleção e triagem da amostra, considerando aspectos como riqueza iconográfica e originalidade; (c) composição das fichas catalográficas; (d) catalogação das imagens e (e) a descrição e interpretação dos resultados.

Assim, adaptando as etapas supracitadas para a presente pesquisa, foi realizado, inicialmente, o mapeamento e registro fotográfico dos estandartes alagoanos a partir de explorações in loco em sedes de agremiações carnavalescas locais e acervos museológicos de cultura popular.

Destaca-se, nessa etapa, a natureza investigativa da pesquisa, já que, considerando a falta de literatura acadêmica voltada à temática dos estandartes carnavalescos no estado de Alagoas, a busca por outras fontes de pesquisas se fez fundamental. Nesse sentido, foram localizadas importantes personalidades do universo carnavalesco alagoano, que contribuíram, por meio de entrevistas não dirigidas, de forma essencial para essa pesquisa. 
Após as duas primeiras etapas, fez-se necessário desenvolver um instrumento de análise capaz de catalogar as imagens e quantificar, de forma eficiente, as características morfológicas dos objetos registrados.

$\mathrm{Na}$ etapa três, intitulada 'composição das fichas catalográficas' foi desenvolvida uma ficha de catalogação que abarcou quatro categorias de classificação e análise dos estandartes (figura 3). A definição dessas categorias se deu de acordo com a classificação de Twyman (2002) para os elementos da LG, a saber: elementos esquemáticos, elementos pictóricos e elementos verbais. Além desses, observou-se a necessidade de se adicionar uma quarta categoria, chamada de dados gerais, destinada a catalogar dados que fogem dos critérios técnicos anteriormente analisados.

Figura 3: Formulários de catalogação das imagens (fontel: autoral)

\section{Seçāo 1 de 4}

Tabulação dos dados colhidos para o trabalho final de graduação

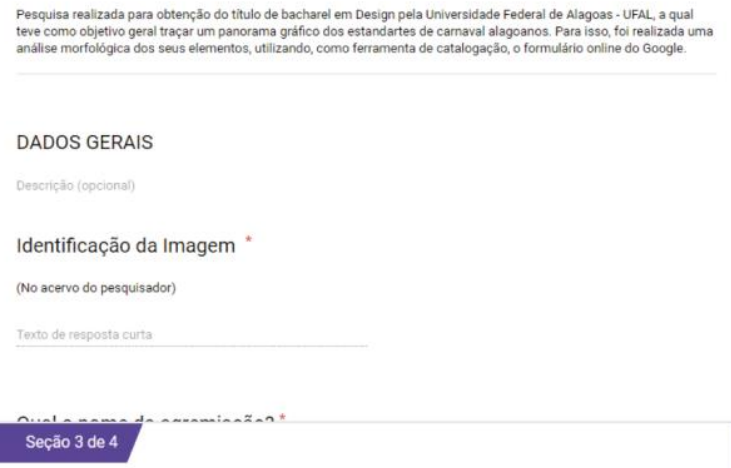

\section{ELEMENTOS PICTÓRICOS}

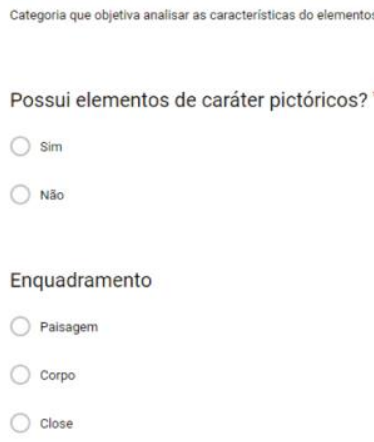

\section{Seçăo 2 de 4}

\section{ELEMENTOS ESQUEMÁTICOS}



\section{ELEMENTOS VERBAIS}

Disposição da letras - movimento *

$\square$ Linea

$\square$ curvelinee

$\square$ Perspective

Disposição das letras - direção

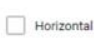

$\square$ vertical

Como ferramenta para auxiliar tal atividade, utilizou-se a plataforma digital do Google Docs que, em suma, permite a elaboração de fichas em forma de questionário, para serem preenchidas online. Após sua aplicação no momento das análises, a ferramenta tabulou as respostas, gerando gráficos automaticamente, facilitando a visualização do universo estudado, pelo pesquisador.

\section{Apresentação e discussão dos resultados}

O corpus dessa pesquisa foi formado por 24 estandartes, associados a blocos de carnaval tradicionais da capital Maceió (figura 4). Os resultados obtidos após a tabulação dos dados permitiu o levantamento de informações interessantes acerca dos aspectos morfológicos dos estandartes alagoanos, além da validação de suposições associadas, empiricamente, a esse universo de pesquisa. 
Figura 4: Nove dos vinte e quarto estandartes catalogados e analisados na pesquisa (fonte: autoral).

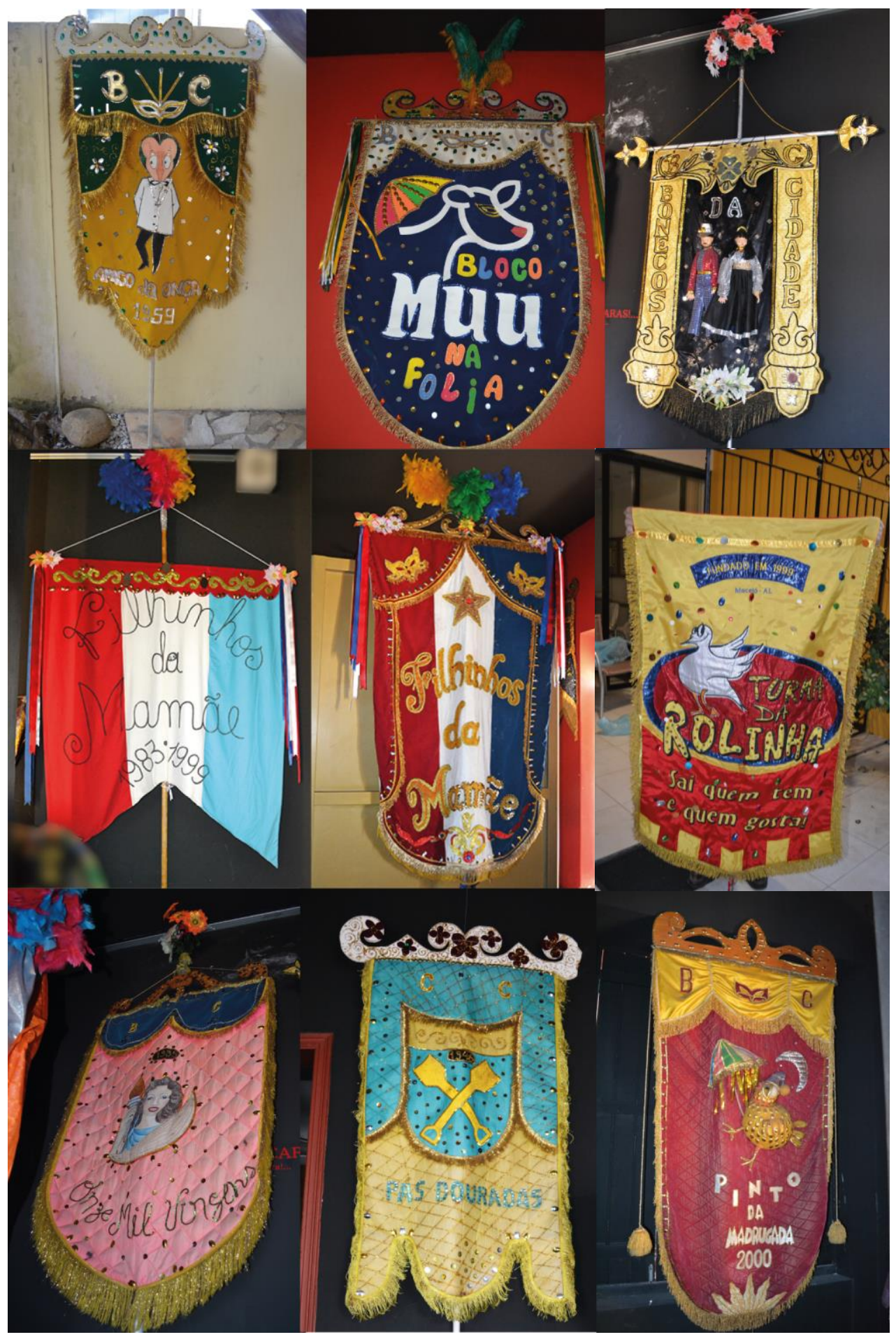

Em dados gerais, foi possível constatar que a catalogação de objetos de produção popular em museus de antropologia contribui diretamente para a preservação da história de um povo, pois, dos 24 artefatos analisados na pesquisa, 17 (70,8\%) deles estão lotados no Museu Théo Brandão.

É possível também perceber que uma diferença significativa dos artefatos quanto à qualidade técnica e modo de produção: estandartes mais antigos apresentam aspectos improvisados, dando pistas de que foram confeccionados por pessoas sem conhecimento artístico - considerados não especialistas. Porém, a maioria dos artefatos - 14 deles $(53,8 \%)-$ 
foram feitos por especialistas na área, utilizando-se de materiais e processos variados como vidrilhos, miçangas e diferentes tecidos.

No que diz respeito aos elementos esquemáticos, em que o principal componente observado foi a própria forma do estandarte, constatou-se uma preferência por estruturas mais tradicionais, com predominância daquelas em formas retangulares com borda inferior arredonda (35\%, referente a 9 artefatos) e dos que possuem apenas duas camadas $(50 \%$, referente a 12 artefatos). Há predileção pelas cores da bandeira de Alagoas, uma das principais representações regionais. Além delas, há uma forte aplicação de elementos metalizados representando as cores prata $(54,2 \%$, referente a 13 artefatos) e, principalmente, dourada (87,5\%, referente a 21 artefatos).

A inserção de elementos pictóricos na composição gráfica dos estandartes tem um grande peso visual, incidindo em $83,3 \%$ da amostra (referente a 20 artefatos). Esses elementos reforçam e ilustram a mensagem verbal presente, onde o nome e o ano de fundação do bloco têm destaque (figura 5).

Figura 5: Estandarte Maracatu Baque Alagoano, no qual o element pictórico tem destaque (fonte: autoral).

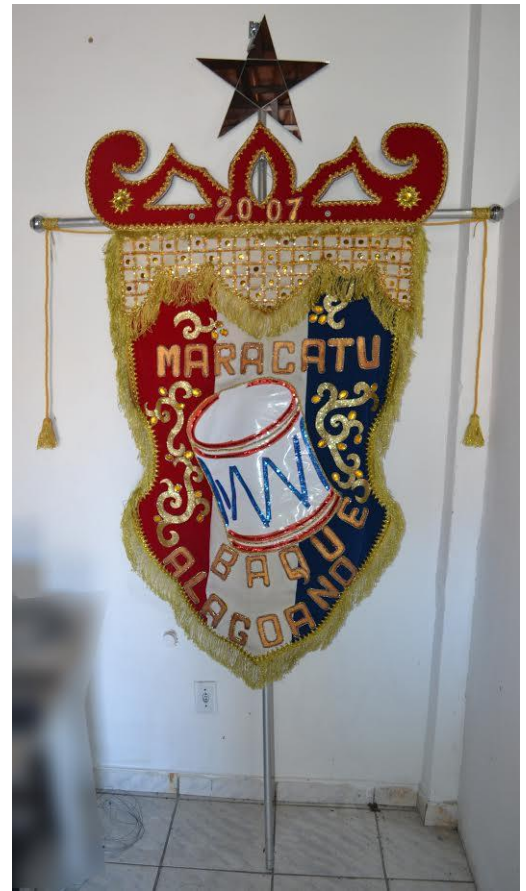

Com relação aos elementos verbais, percebeu-se que existe, em praticamente todas as categorias, um tipo de configuração que sempre se destaca em relação às outras. Dentre elas, temos o direcionamento linear, presente em $66,7 \%$ da amostra (referente a 16 artefatos), a orientação horizontal presente em $87,5 \%$ da amostra (referente a 21 artefatos) e o alinhamento centralizado presente em $79,2 \%$ da amostra (referente a 19 artefatos). Essa conclusão reforça suposições de que, apesar dos estandartes apresentarem uma variada e rica gama gráfica, é possível observar padrões morfológicos na composição dos seus elementos verbais.

Ainda nessa categoria, um critério de grande representatividade foi o tipo de letra adotado, no qual, dentre nove classificações tipográficas vernaculares possíveis (HENNES, 2012), a letra gorda se sobressai com incidência em $41 \%$ da amostra (referente a 10 artefatos). As letras expressivas e fantasia não foram registradas.

\section{Considerações finais}

Os dados obtidos, a partir das análises registradas, permitiu-nos perceber e reconhecer esses estandartes sob uma nova perspectiva, buscando a percepção dos artefatos como objetos culturais, passíveis de serem analisados sob a ótica do design da informação. Essa análise contemplou desde os modos de produção desses artefatos, aos materiais utilizados na sua 
confecção. Foi possível também descobrir peculiaridades plásticas e visuais que formam padrões gráficos, bem como particularidades inexploradas que fazem parte desse universo.

Como reflexão para futuras pesquisas, ressalta-se a área do design da informação como vasto campo acadêmico, capaz de contribuir para o reconhecimento e valorização das produções artesanais de artefatos culturais, visando auxiliar a perpetuação da tradição, identidade e memória gráfica dos artefatos populares.

\section{Referências}

CAVALCANTI, B. C. (2014). Muitos Carnavais. (J. Ávila, entrevistador)

DONDIS, D. A. (2003). Sintaxe da Linguagem Visual. São Paulo: Martins Fontes.

FARIA, P. C., \& SOUTO, V. T. (2014). Linguagem gráfica de infográficos online do governo brasileiro - Um estudo de caso do Portal Brasil. Revista Brasileira de Design da Informação, 320-336.

HENNES, M. (2012). Letreiros Populares do Recife. Uma análise dos seus aspectos semâticos e morfológicos. Dissertação de mestrado não publicada. Recife: Universidade Federal de Pernambuco.

SILVA, L. D. (17 de Setembro de 2016). Porta-estandarte, presença medieval no carnaval de Pernambuco. In: Revivendo Músicas.

<http://revivendomusicas.com.br/curiosidades_01.asp?id=138> 20/04/2017

TWYMAN, M. L. 2002. Further thoughts on a schema for describing graphic language.

Proceedings of the 1st International Conference on Typography and Visual Communication, 329-350. Thessaloniki, Greece: University of Macedonia Press.

\section{Sobre o(a/s) autor(a/es)}

Felipe Dias Albuquerque, BSc, UFAL, Brazil <albuquerque.felipedias@gmail.com>

Mariana Hennes, MSc, UFAL, Brazil <mhennes.design@gmail.com> 